NBI-HE-91-32

August 27, 1991

\title{
MEASURING THE STRING TENSION IN RANDOM SURFACE MODELS WITH EXTRINSIC CURVATURE
}

\author{
A. Irbäck \\ CERN, Theory Division, CH 1211 Geneva 23, Switzerland \\ J. Jurkiewicz ${ }^{1}$ and $S$. Varsted \\ The Niels Bohr Institute \\ Blegdamsvej 17, DK-2100 Copenhagen $\varnothing$, Denmark
}

\begin{abstract}
A highly vectorized fortran program for Monte Carlo simulation of dynamical triangulated random surfaces with extrinsic curvature is presented. It is an implementation of a new procedure to measure the string tension. We summarize the method and give a detailed description of the program.
\end{abstract}

\footnotetext{
${ }^{1}$ Permanent address : Inst. of Phys., Jagellonian Univ., ul. Reymonta 4, PL-30 059 Kraków 16, Poland
} 


\section{PROGRAM SUMMARY}

\section{Title of program: STRTEN}

Program obtainable from:

nirback@cernvm.bitnet

Computer: Cray Y-MP. Easily portable to any vector computer with efficient treatment of short vectors.

\section{Operating system: UNICOS}

\section{Programming language used: FORTRAN}

High speed storage required: 2.6 MWords for a simulation of 64 systems with 400 points each.

No. of bits in a word: 64

Peripherals used: reads/writes configuration and data from/to disk.

No. of lines in combined program and test deck: 1540

Keywords: random surfaces, dynamical triangulation, extrinsic curvature, Monte Carlo, strings, string tension, vectorization.

Nature of the physical problem:

The dynamical triangulated random surface with extrinsic curvature is investigated, with emphasis on its string tension.

\section{Method of solution:}

The program STRTEN performs a Monte Carlo simulation of the model. The dynamical nature of the triangulation restricts the possibilities to vectorize such a program. In STRTEN vectorization is achieved by simulating a number of independent systems in parallel, a method first described in Ref. [1]. In this way we obtain a speedup factor of 6 between vector and scalar mode.

The string tension is extracted by using the method in Ref. [2]. This means that we consider systems with the topology of a torus and with twisted boundary conditions in the embedding space. For a fixed number of points in the triangulation, the following quantities are measured:

- The mean value of the gaussian part of the action.

- The mean area of a triangle in the triangulation.

- The mean value of the extrinsic curvature part of the action, which is taken to be the sum of the cosines of the angles between the normals to two neighbouring triangles.

- The mean extent of the surface.

- The susceptibility.

The string tension is obtained from the gaussian term.

The embedding space is assumed to be $d=3$-dimensional, but the modifications required for arbitrary $d$ are moderate.

Typical running times: The time required for an attempt to update three links and the coordinates at one vertex is $17.2 \mu$ seconds. The time needed for measurements is negligible.

\section{References:}

1. J. Ambjørn, D. Boulatov and V.A. Kazakov, Mod. Phys. Lett. A5 (1990) 771.

2. J. Ambjørn, J. Jurkiewicz, S. Varsted, A. Irbäck and B. Petersson, "Critical properties of the dynamical random surface with extrinsic curvature", preprint NBI-HE-9114, May 1991. 


\section{Introduction}

In the search for a discretized version of a bosonic string theory, the model of triangulated random surfaces with gaussian action was proposed in 1985 [1]. It was hoped that this formulation could be used to understand the properties of a bosonic string in sub-critical dimensions $d<26$. Investigations carried out over the past few years seem, however, to indicate that for any $d \geq 1$ it degenerates into a theory of branched polymers.

To overcome this problem it was suggested to add to the action a new term, proportional to the extrinsic curvature, which would smoothen the surface and possibly avoid the collapse into a branched polymer. On a triangulated surface various forms of this term have been investigated [2], but it seems that only the one proposed in [3] leads to a consistent theory of surfaces. The theory with an action modified in this way appears to have a second order phase transition at a non-zero value of the coupling constant of the extrinsic curvature term. The scaling behaviour of the string tension near this critical point was studied numerically in Ref.[4]. The system considered in this investigation had the topology of a disc and a fixed boundary. A fixed number of points were assigned both to the interior and to the boundary. The values extracted measured only the change of the string tension relative to its critical value.

The program presented in this paper implements a method to obtain a direct measurement of the string tension [5]. The formulation has the additional advantage that it avoids possible systematic errors connected with the introduction of a fixed boundary. The new method uses a system with the topology of a torus and with twisted boundary conditions in target space. The string tension is obtained as a derivative with respect to the twist parameter. By a simple rescaling of the coordinates, one can express this derivative in terms of the gaussian part of the action.

The program is designed to run efficiently on a vector computer. To achieve this we simulate a number of independent systems in parallel. The optimal choice for the number of systems to be simulated depends, of course, both on the computer used and the parameters studied. If a large portion of the computer time has to be spent on thermalizing the system, it is clear that it can be advantageous to simulate just a single system. To get acceptable statistics one often needs, however, to perform runs which are much longer than the thermalization time. It then becomes worthwhile to simulate several systems in parallel. In our calculations on the Cray Y-MP we have been using 64 independent systems. The speedup obtained between vector and scalar mode was a factor of 6 .

\section{Boundary conditions}

The model is inspired by Polyakov's formulation of the bosonic string theory with the triangulation playing the role of the metric, and is expected to belong to the same universality class as the ones studied in [4]. Letting $\langle i j\rangle$ denote a link from point $i$ to point $j$ in the triangulation, the action is given by

$$
A\left(\vec{X}_{i j}, T\right)=A_{G}\left(\vec{X}_{i j}, T\right)+\lambda A_{C}\left(\vec{X}_{i j}, T\right)=\frac{1}{2} \sum_{\langle i j\rangle} \vec{X}_{i j}^{2}+\lambda \sum_{\langle i j\rangle}\left(1-\cos \theta_{i j}\right)
$$


with $\theta_{i j}$ being the angle between the normals to the triangles $\langle i j k\rangle$ and $\langle j i l\rangle$ with a common link $\langle i j\rangle . \vec{X}_{i j}$ denotes a vector in the $d$-dimensional enveloping Euclidean space, with the three vectors forming a triangle $\langle i j k\rangle$ satisfying

$$
\vec{X}_{i j}+\vec{X}_{j k}+\vec{X}_{k i}=0
$$

The vectors $\vec{X}_{i j}$ can be simply related to the positions $\vec{X}_{i}$ and $\vec{X}_{j}$ the points $i$ and $j$ in the enveloping space.

For a system with a topology of a disc the string tension $\sigma$ is defined by the asymptotic behaviour of the partition function

$$
Z(S)=\sum_{T \in \mathcal{T}} e^{-\beta|T|} \int\left[\prod_{i \in T, \partial T=\partial S} \mathrm{~d}^{d} X_{i}\right] \delta\left(\sum_{i} \vec{X}_{i}\right) e^{-A\left(\vec{X}_{i j}, T\right)},
$$

where $S$ is a flat two-dimensional surface with area denoted by $a(S)$. In the limit $a(S) \rightarrow$ $\infty$, one defines

$$
e^{-a(S) \sigma} \sim Z(S)
$$

In Eq.(3) one sums over all random surfaces with a boundary fixed to that of a flat surface with area $a(S)$. This grand canonical definition is very difficult to use in numerical simulations. In practice the behaviour of the string tension is deduced from simulations of the canonical partition function with a fixed number $|T|$ of triangles. In previous work some of the points of the lattice were fixed on a boundary to ensure the condition $\partial T=\partial S$.

In order to let the triangulations vary more freely at the boundary we have chosen to do the simulations using a system with the topology of a torus, but with nontrivial boundary conditions. These conditions amount to introducing a surface $S$ shaped like a rectangle with sides $y_{1}$ and $y_{2}$ and with periodic boundary conditions for the triangulation. This means that we average over infinitely many replicas of the system, each with the same flat triangulation, but with $\vec{X}_{i}$ 's that differ by an integer combination of the vectors $\vec{E}_{1}=\left(y_{1}, 0,0, \ldots\right)$ and $\vec{E}_{2}=\left(0, y_{2}, 0, \ldots\right)$ which make up the sides of $S$. The different replicas are sewed together by the triangulation and they have, of course, the same value of the action $A$.

The partition function is expressed in terms of the vertex variables $\vec{X}_{i}$ belonging to one of the replicas. Its dependence on the boundary conditions enters through the relation between the $\vec{X}_{i}$ 's and the link variables $\vec{X}_{i j}$. Let us discuss this relation in some detail. In the case of periodic boundary conditions in target space, $y_{1}=y_{2}=0$, we can write $\vec{X}_{i j}=\vec{X}_{j}-\vec{X}_{i}$. With nontrivial boundary conditions this is of course impossible. Consider the sum of $\vec{X}_{i j}$ 's along an arbitrary closed loop $C$ in the triangulation. In the general case the boundary conditions imply that $\sum_{C} \vec{X}_{i j}=n_{1}^{C} \vec{E}_{1}+n_{2}^{C} \vec{E}_{2}$, where the integers $n_{1}^{C}$ and $n_{2}^{C}$ count, with sign, how many times the loop winds around each axis of the torus. To describe this situation we introduce a link variable $\vec{E}_{i j}$ associated with the triangulation. We demand that $\vec{E}_{i j}+\vec{E}_{j k}+\vec{E}_{k i}=0$ for every triangle $\langle i j k\rangle$. We can then write

$$
\vec{X}_{i j}=\vec{X}_{j}-\vec{X}_{i}+\vec{E}_{i j},
$$

provided that

$$
\sum_{C} \vec{E}_{i j}=n_{1}^{C} \vec{E}_{1}+n_{2}^{C} \vec{E}_{2}
$$



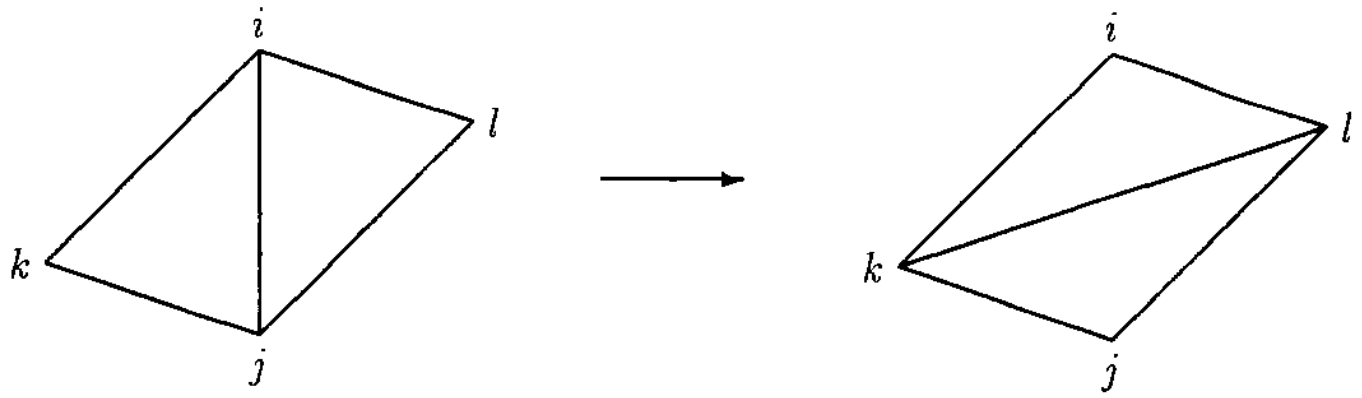

Figure 1: The flip procedure.

for every closed loop $C$. Using this decomposition, we define the partition function

$$
Z(y)=\sum_{T \in \mathcal{T}} e^{-\beta|T|} \int\left[\prod_{i \in T} \mathrm{~d}^{d} X_{i}\right] \delta\left(\sum_{i} \vec{X}_{i}\right) e^{-A\left(\vec{X}_{i j}, T\right)}
$$

where the $y$ dependence is through the dependence of $\vec{X}_{i j}$ on $\vec{E}_{i j}$. The condition Eq.(6) does not uniquely specify the $\vec{E}_{i j}$ 's associated with a triangulation $T$, but is sufficient to make the contribution to the partition function well-defined. This is easily seen by using the transformation

$$
\begin{aligned}
\vec{X}_{i} & \rightarrow \vec{X}_{i}+l_{i}^{1} \vec{E}_{1}+l_{i}^{2} \vec{E}_{2}, \\
\vec{E}_{i j} & \rightarrow \vec{E}_{i j}+l_{i}^{1} \vec{E}_{1}+l_{i}^{2} \vec{E}_{2}-l_{j}^{1} \vec{E}_{1}-l_{j}^{2} \vec{E}_{2},
\end{aligned}
$$

which leaves the vectors $\vec{X}_{i j}$ invariant.

In a numerical simulation it is in principle sufficient to keep track of the variables $\vec{X}_{i j}$. They contain all the information necessary. Such a representation leads, however, to an uncontrollable accumulation of rounding errors, which, in particular, can cause violations of the boundary conditions initially imposed. To avoid this problem we store separately the variables $\vec{X}_{i}$ and $\vec{E}_{i j}$ in the decomposition Eq.(5). It is clear that we can always chose $\vec{E}_{i j}$ to be of the form $\vec{E}_{i j}=n_{i j}^{1} \vec{E}_{1}+n_{i j}^{2} \vec{E}_{2}$ with $n_{i j}^{1}$ and $n_{i j}^{2}$ integers. This choice makes it easy to prevent rounding problems. The decomposition is however not yet uniquely specified. With integer valued $l_{i}^{1}$ and $l_{i}^{2}$ one can still perform the transformation in Eq.(8). This additional freedom can be used to keep the vectors $\vec{E}_{i j}$ bounded.

After initialization the simulation can be performed by using standard update schemes. The update procedure for the triangulation is the well-known flip (see Figure 1) which is known to be ergodic in the class of all triangulations with the same topology [6]. As usual we restrict the sum over all triangulations to those with no two-loops and no points with less than three neighbours. This is done by forbidding flips that lead to such triangulations. At a flip the $\vec{E}_{i j}$ 's have to be changed, while the $\vec{X}_{i}$ 's remain the same.

Next we turn to the update of the coordinates $\vec{X}_{i}$. Note that an update of the coordinates does not affect the nontrivial boundary conditions. The simplest way is to add to $\vec{X}_{i}$ a random shift taken from an appropriate distribution. The new configuration is then accepted/rejected with probability $P_{a c c}=\min (1, \exp (-\Delta A))$. We have also tested two modifications of this Metropolis scheme [7] in which the new values are proposed in a different way. The modified methods are obtained by performing a heatbath and an $\omega=2$ overrelaxation [8] update, respectively, with respect to the simple gaussian part of 
the action. To fulfil detailed balance one then has to accept/reject the new values with probability $P_{a c c}=\min \left(1, \exp \left(-\lambda \Delta A_{C}\right)\right)$, where $\Delta A_{C}$ is the change in the extrinsic curvature term. In these two schemes, with $\omega$ fixed in the latter, there is no free parameter which can be used to tune the acceptance rate. Beforehand, it is thus not clear that a reasonable acceptance can be obtained. We tested a few combinations of the three methods mentioned at parameters $\lambda=1.5$ and $r=1 / 16$ for $N=36$ and 64 . The acceptance rate for the modified methods turned out to be fairly low, roughly $20 \%$. Autocorrelation times were nevertheless somewhat smaller than those we had observed using random shifts only. Using heatbath and overrelaxation, each in half of the cases, to find the new values, we observed autocorrelations which were a factor 0.6 smaller than with random shifts only. This reduction factor did not change significantly with $\mathrm{N}$, nor did the acceptance rate for the modified updates. In the program all the three updates mentioned are available. The relative frequencies with which they are performed are specified through parameters.

\section{Measurement}

In order to extract the string tension from a simulation we have to make the the connection between the quantity $\mathcal{G}\left(y_{1}, y_{2} ; \beta\right)=-\log Z\left(y_{1}, y_{2}\right)$ from the grand canonical ensemble and the free energy $\mathcal{F}\left(y_{1}, y_{2} ; N\right)=-\log Z_{N}\left(y_{1}, y_{2}\right)$ expressed through the canonical partition function,

$$
Z_{N}\left(y_{1}, y_{2}\right)=\sum_{T \in \mathcal{T},|T|=N} \int\left[\prod_{i \in T} \mathrm{~d}^{d} X_{i}\right] \delta\left(\sum_{i} \vec{X}_{i}\right) e^{-A\left(\vec{X}_{i}, y_{1}, y_{2}, T\right)}
$$

These two are related by a Legendre transform

$$
\mathcal{G}\left(y_{1}, y_{2} ; \beta\right)=N \beta+\mathcal{F}\left(y_{1}, y_{2} ; N\right),
$$

with $N=\partial \mathcal{G} / \partial \beta$. In Eq.(9) the dependence of the action on $\vec{X}_{i}, y_{1}$ and $y_{2}$ is through the $\vec{X}_{i j}$ 's, as discussed in the previous section. Using simple scaling arguments [5] we can relate the string tension $\sigma$ to the derivative

$$
\bar{\sigma}(S / N)=\partial \mathcal{F} / \partial S
$$

where $S=y_{1} y_{2}$. This quantity can be easily obtained from (9). Let us consider here the simplest case $y_{1}=y_{2}=y$, which can trivially be generalized to arbitrary values of $y_{i}$.

Differentiate $\log Z_{N}(y)$ with respect to $y$. This can be done by scaling all lengths in $Z_{N}(S)$ by $1 / y$ and pulling out a factor of $y^{2}$ in front of the gaussian part of the action

$$
\begin{aligned}
Z_{N}(y)= & y^{d(N-1)} \sum_{T \in \mathcal{T},|T|=N} \\
& \int\left[\prod_{i \in T} \mathrm{~d}^{d} X_{i}\right] \delta\left(\sum_{i} \vec{X}_{i}\right) \exp \left[-y^{2} A_{G}\left(\vec{X}_{i}, y=1, T\right)-\lambda A_{C}\left(\vec{X}_{i}, y=1, T\right)\right]
\end{aligned}
$$

Here the differentiation can be explicitly done, and after a scaling by $y$ we get

$$
\frac{\partial \log Z_{N}(y)}{\partial y}=\frac{d(N-1)-2\left\langle A_{G}(y, T)\right\rangle}{y}
$$


giving the string tension

$$
\bar{\sigma}=\frac{\left\langle A_{G}(y, T)\right\rangle-d(N-1) / 2}{y^{2}}
$$

So all we have to do is to measure the expectation value of the gaussian part of the action for a set of values of $y$ and $N$ and insert it in this formula, to get a number of values $\vec{\sigma}$. If these values turn out to be dependent on $y$ and $N$ through $y^{2} / N$ in a certain scaling window, we can relate the measured quantity to the physical string tension [5].

\section{Program description}

As we explained in the abstract the vectorization of the program is achieved through simulating a number wSYS systems in parallel (we use NSYS $=64$ for the Cray). These systems are treated completely independently. All observables are measured in each system to get NSYS statistically independent values, from which averages and errors can be estimated.

The shape of the surface is given by the vectors $\vec{X}_{i j}$, which are stored in the array RL. They contain, for a given triangulation, all the information necessary. In addition we use, as discussed in Section 2, the decomposition Eq.(5), which enables us to control rounding errors. All the updates are thus performed on the vectors $\vec{X}_{i}$ and $\vec{E}_{i j}$ and the $\vec{X}_{i j}$ 's are then computed using Eq.(5). Further quantities, which are used in the calculation of the action, are stored in order to save computer time. Initialization of the coordinates and the triangulation takes place in the subroutine ronus. The triangulation is described by the pointers LINK, POINT and PLINK. A complete list of the variables used to describe the system is given below. The list also includes the corresponding common-blocks. Unlike the other parameters listed, the dimension NDIM of the embedding space is not a free one. For optimization purposes we have assumed a fixed value NDIM $=3$ in many of the subroutines. The modifications required for NDIM $\neq 3$ are however moderate.

All points and links carry numbers. Links are considered oriented so that the number of the link $\langle i j\rangle$ is minus the number of the link $\langle j i\rangle$.

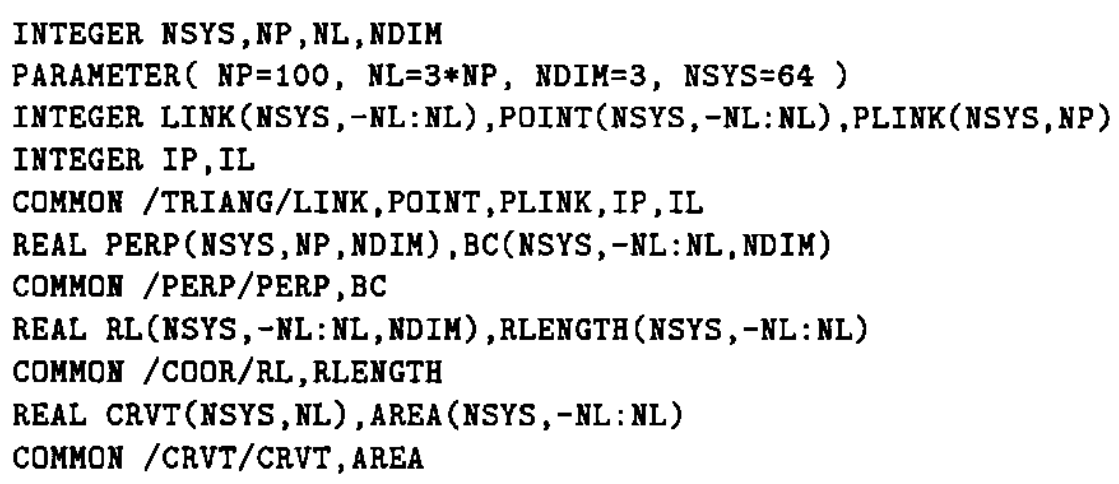

In the following $\mathrm{L}$ is the number of the link $\langle i j\rangle$.

NP The maximal number of points in each triangulation.

NL The maximal number of links in each triangulation.

NDIM The dimension of the embedding space.

wsYS The number of systems simulated in parallel. 

LINK (ISYS, L) is the number of the link found going clockwise around the point $i$ in system
ISYS.

POINT(ISYS, L) is $j$.

PLINK(ISYS, I) holds a link that satisfies POINT(ISYS, -PLINK(ISYS, I)) =I.

IP The current number of points in each triangulation.

IL The current number of links in each triangulation.

PERP (ISYS, I, J) is the $\mu=\mathrm{J}$ component at site $i=\mathrm{I}$ of $X_{i}^{\mu}$.

$\mathrm{BC}$ (ISYS, L, I) is the $\mu=\mathrm{I}$ component of $E_{i j}^{\mu}$.

RL(ISYS, L, I) is the $\mu=\mathrm{I}$ component of $X_{i j}^{\mu}$.

RLENGT (ISYS, ABS(L)) is $\vec{X}_{i j} \cdot \vec{X}_{i j}$.

RLENGT (ISYS, $-A B S(L)$ ) is the square of the distance between the two points lying next to the link $\mathrm{L}$.

CRVT (ISYS, ABS(L)) is the cosine of the angle between the two triangles next to $L$.

$\operatorname{AREA}($ ISYS,$L$ ) is twice the area of the triangle next to $L$ in clockwise direction around $i$.

AREA (ISYS, L) is twice the area of the triangle next to $L$ in clockwise direction around $j$.

The program is built around the two procedures SHIFT and FLIP which together with their subroutines perform the Monte Carlo updates of the systems.

The subroutine SHIFT attempts to update the vectors $\vec{X}_{i}$ at one randomly chosen vertex in each of the NSYS systems. New values are proposed by using one of the three methods described in Section 2. Heatbath and overrelaxation with respect to the gaussian part of the action are used for a fraction FRHB and FROR, respectively, of the calls to SHIFT. The third possibility available is to add to the $\vec{X}_{i}$ 's a vector with components chosen with uniform probability from the interval $[-\mathrm{RC} / 2, \mathrm{RC} / 2]$. The suggested updates are then accepted/rejected in a Metropolis step.

The update of the triangulation is done in a similar way. One randomly chosen link from each system is considered.The subroutine NEILIN checks if the new triangulation fulfils the conditions mentioned in Section 2.

A problem repeatedly encountered in the update procedures is to find all the neighbours to a given vertex. Because of the dynamical nature of the triangulation some care has to be taken. Figure 2 shows a typical example of how it is done in the program.

There are three special subroutines dealing with potential numerical problems. In subroutine GT the transformation Eq.(8) is used to prevent the vectors $\vec{E}_{i j}$ from becoming too large. The subroutine casts them into the form $\vec{E}_{i j}=n_{i j}^{1} \vec{E}_{1}+n_{i j}^{2} \vec{E}_{2}$ with $n_{i j}^{1,2}=0$ or \pm 1 . After having done that, it is easy to check that rounding errors have not become unacceptably large. This check is performed in subroutine RENORM. Finally, the subroutine CM adjusts the position of the center of mass $\sum_{i} \vec{X}_{i} / N$. These three subroutines are called with a frequency specified through the variable IREN. In our calculations we found that IREN $=1000$ was sufficient to keep rounding errors negligible.

Measurements are separated by IMEAS Monte Carlo sweeps and are performed in the subroutine MEASUR. Since all the variables listed above are updated during the Monte Carlo iterations, most of the measurements are trivial. Five quantities are written to the data 
file for later analysis. These are the gaussian and extrinsic curvature parts of the action, the average area of a triangle, the extent of the surface and the susceptibility. As a measure of the extent of the surface we take

$$
\frac{1}{N} \sum_{i} \sqrt{\sum_{\mu}^{\prime} X_{i}^{\mu_{2}}},
$$

where the sum over $\mu$ runs over directions with strictly periodic boundary conditions. If, for instance, $y_{1}$ and $y_{2}$ are both non-zero, then the $\mu=1$ and 2 terms are omitted. The susceptibility we define as $\left[N^{-1} \sum_{\langle i j k\rangle} \hat{n}_{i j k}\right]^{2}$, where $\hat{n}_{i j k}$ is the normal to the triangle $\langle i j k\rangle$.

The standard output file contains averages over bins of NWRITE measurements. Acceptance rates for the flips and the shifts are also given.

\section{Summary}

We have presented a program to simulate dynamical triangulated random surfaces with an extrinsic curvature term in the action. The program implements a new procedure to make an absolute measurement of the string tension, which we have described briefly. A more detailed description of the method can be found in Ref.[5], which also contains numerical results obtained with the program. To take advantage of the speed of vector computers we have written a program which simultaneously simulates several independent systerns. We have shown that in this way a significant speedup can be obtained, despite of the irregularity of the problem. 


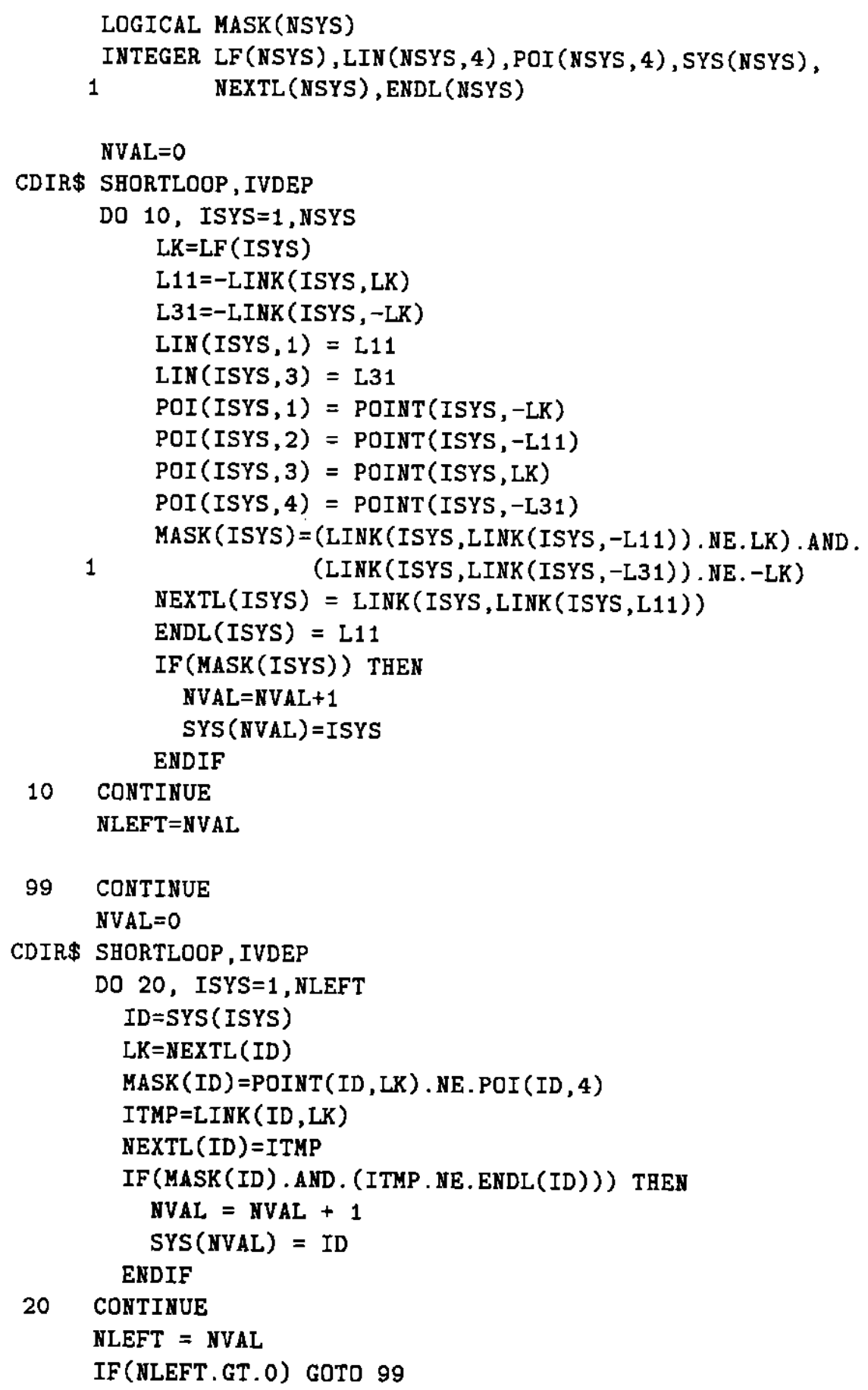

Figure 2: Part of the subroutine NEILIN, which is used to check whether or not the proposed flips, of the links contained in LF(ISYS), are allowed. The first part checks that the two points connected by the link to be removed both have at least four neighbours. The second part checks that the two points to be connected by the new link are not already neighbours. This second part involves a search for the neighbours of a given point, POI(ISYS, 2). After execution of the code MASK (ISYS) will be .FALSE. if not both the conditions are fulfilled. 


\section{References}

[1] J. Ambjørn, B. Durhuus, and J. Frölich, Nucl. Phys. B257 (1985) 433;

F. David, Nucl. Phys. B257 (1985) 543;

V.A. Kazakov, I.K. Kostov, and A.A. Migdal, Phys. Lett. 157B (1985) 295.

[2] Y. Kantor and D.R. Nelson, Phys. Rev. Lett. 58 (1987) 2774;

J. Ambjørn, B. Durhuus and T. Jonsson, Nucl. Phys. B316 (1989) 526;

M. Baig, D. Espriu and J.F. Wheater, Nucl. Phys. B316 (1989) 587;

J. Kogut, A. Moreo and S. Das, Nucl. Phys. B327 (1989) 530.

[3] J. Ambjørn, B. Durhuus, J. Frölich and T. Jonsson, Nucl. Phys. B290[FS20] (1987) 480.

[4] S.M. Caterrall, Phys. Lett. B243 (1990) 121.

[5] J. Ambjørn, J. Jurkiewicz, S. Varsted, A. Irbäck and B. Petersson, Critical properties of the dynamical random surface with extrinsic curvature, preprint NBI-HE-91-14, May 1991, to appear in Phys. Lett. B.

[6] D.V. Boulatov, V.A. Kazakov, I.K. Kostov, A.A. Migdal, Nucl. Phys. B275 (1986) 641.

[7] N. Metropolis, A.W. Rosenbluth, M.N. Rosenbluth, A.H. Teller and E. Teller, J. Chem. Phys. 21 (1953) 1087.

[8] S.L. Adler, Phys. Rev. D23 (1981) 2901. 